\title{
Structural Study of Acetylene Films on Exfoliated Graphite
}

\author{
M. Trabelsi ${ }^{1 *}$, F. Khémiri ${ }^{1}$, S. Missaoui ${ }^{1}$, H. Ettayeb ${ }^{1}$, J. P. Coulomb ${ }^{2}$, Y. Larher $^{3}$ \\ ${ }^{1}$ Laboratoire de physique des matériaux : Propriétés et Applications, Faculté des Sciences de Bizerte, 7021 Jarzouna Bizerte, Tunisie \\ ${ }^{2}$ CINaM/ CNRS, Compus de Luminy, 13288 Marseille, Cedx 9, France \\ ${ }^{3}$ DRECAM, CEA Saclay, Gif sur Yvette France
}

\begin{abstract}
The structures of deuterated acetylene $\left[C_{2} D_{2}\right]$ thin films adsorbed on the (0001) surfaces of exfoliated graphite have been investigated by elastic neutron diffusion. The aim of this study was to assess the structures of the condensed phases. Neutron diffraction spectra have been recorded at temperature ranging from $10 \mathrm{~K}$ to $141 \mathrm{~K}$ and for coverages corresponding to the monolayer completion 0.7 layer $\leq \theta \leq 1.23$ layers. Two bidimensional solid phases $S_{1}$ and $S_{2}$ have been identified. Their structures present commensurability with the (0001) graphite surface. At an intermediate coverage $(\theta=1.0$ layer) there is coexistence of the two solid phases. The evolution of the recorded spectra with the temperature suggests the fusion of the $S_{1}$ solid to occur at $T_{m}\left(S_{1}\right)=135 \pm 1 K$. The fusion of $S_{2}$ solid happened at higher temperature: $T_{m}\left(S_{2}\right)>T_{m}\left(S_{1}\right)$.
\end{abstract}

Keywords: neutron diffraction, physisorbed phases, commensurate phases

\section{Introduction}

Graphite, with its well defined and uniform surface, is still considered as a basic model for investigating adsorption mechanisms on carbonaceous substrates. In fact, a sheet of carbon atoms arranged in a hexagonal framework constitute the principal piece of graphene or carbon nanotubes. These later are known do to their scientific and technological interest and do to the particular properties they induce within sorbed phases. Besides noble gases and simple molecules, the adsorption of hydrocarbon ones on uniform substrates has been widely undertaken [1-8].

It has been inferred that the structures of alkanes molecules investigated at sub-monolayer coverage's and low temperature are isomorphous with rectangular unit cells of plane group pgg containing two molecules per cell [1].

Many studies have been devoted to the adsorption of acetylene on uniform surface substrates. If we limited our bibliographic study to graphite, $\mathrm{MgO}$ and $\mathrm{NaCl}$ as substrates we can quote [9-19] among a large list. However, only three groups attempted to determine the structures of condensed phases of $\mathrm{C}_{2} \mathrm{H}_{2}$ on graphite [13-15].

In the first study [13], Menaucourt \& al. have measured adsorption isotherms of $\mathrm{C}_{2} \mathrm{H}_{2}$ on exfolieted graphite in the temperature range $110 \mathrm{~K}-160 \mathrm{~K}$. Based on the area occupied by a $\mathrm{C}_{2} \mathrm{H}_{2}$ molecule $\sigma$ when adsorbed on graphite and at the various steps observed in an isotherm and by comparing $\sigma$ to the surface of one molecule determined from Van Der Waals radius, they conclude about the existence of three (2D) dense phases. But they have no sufficient matter to establish these structures.

In the second one [14], Thorel \& al. have measured neutron diffraction spectra by the film of acetylene adsorbed on graphite at low temperature $\mathrm{T}=57 \mathrm{~K}$. Two bidimensional (2D) solid phases have been reported respectively $S_{1}$ for the coverage 0.65 to 0.89 layers and $S_{2}$ for the coverage 1.24 to 1.54 layers. At a coverage corresponding to 0.99 layer, the measured spectrum was attributed to a mixture of the previous $S_{1}$ and $S_{2}$ phases.

In the last study [15], Peters \& al. have measured adsorption isotherms of $\mathrm{C}_{2} \mathrm{H}_{2}$ on graphite at the temperature range 109 to $184 \mathrm{~K}$. But since they used a compressed graphite as a substrate, their conclusion about the (2D) critical temperature $T_{C}(2 D)$ was incorrect and this point has been discussed elsewhere [16]. Concerning the structures of the (2D) solid phases, they tried to reproduce the neutron diffraction spectra by acetylene adsorbed on graphite and presented in [14]. Using Monte Carlo simulations, their investigation has improved the structures of the $S_{1}$ and $S_{2}$ solid phases by proposing for each one of the two (2D) solids a unit cell containing two molecules instead of those containing one molecule and proposed by Thorel [14].

Firstly, to complete a previous paper [18] where thermodynamic characterisation of acetylene thin films adsorbed on graphite has been exposed, and secondly to assess the structures of the two (2D) dense phases observed at the completion of the monolayer, we present here a detailed study of the structural investigation of $\mathrm{C}_{2} \mathrm{D}_{2}$ adsorbed on exfoliated graphite. The structures retained for the two bidimensional solid phases were supported by the thermodynamic characterization mentioned above.

\section{Experimental Techniques}

Neutron diffraction spectra by the two-dimensional acetylene phases condensed on graphite were measured at the Atomic Study Center in Saclay, France. We used the instrument G6-1 equipped with a multi-detector formed of 400 cells and covering an angular range $2 \theta=80^{\circ}$, as well as a cryostat allowing to regulate between $2 \mathrm{~K}$ and $300 \mathrm{~K}$ to better than $0.05 \mathrm{~K}$, and on rather long times (the counting of a spectrum can last up to 12 hours), the wavelength used is $\lambda$ 
$=4.725 \AA$. The spectra giving the intensity (number of hits) as a function of the angle are recorded during the manipulation.

In this type of manipulation, a background noise spectrum is recorded (bare substrate), then spectra (substrate + adsorbate) are recorded, the spectra corresponding to the condensed phase are therefore difference spectra. So that the signal due to the condensed matter is sufficiently intense and as in all neutron diffraction experiments we used deuterated acetylene $\mathrm{C}_{2} \mathrm{D}_{2}$ because the coherent diffusion coefficient of deuterium is greater than that of hydrogen.

In such experiments, the structural parameters $(a, b, \gamma)$ of the unit cell of the (2D) solid phase are deduced from the peaks positions, whereas the peaks intensities are correlated to the orientation of the molecules in the unit cell. on the other hand, the width at mid-height of a peak of Miller indices hk is an indication of the extent of the order in the direction hk i.e. short range order or long range order

In what follows, we first give the spectra measured as a function of the recovery rate by keeping the temperature constant and from which we have determined the structures of the different condensed phases.
Then we will present the evolution of the neutron diffraction spectra by the determined (2D) solids as a function of temperature in order to locate their melting temperature and / or loss of the orientational order within.

\section{Results and Discussion}

Neutron diffraction spectra have been recorded for coverages 0.7 layer $\leq \theta \leq 1.23$ layer at temperatures $10 \mathrm{~K} \leq \mathrm{T}$ $\leq 141 \mathrm{~K}$. In figure-1 are shown the spectra measured at $\mathrm{T}=121 \mathrm{~K}$ for coverages $\theta_{1}=0.7$ layer; $\theta_{2}=1.1$ layer and at an intermediate coverage $\theta_{12}=1.0$ layer. Such spectra are difference ones (with and without adsorbate). The saw-tooth line shape of the observed peaks is the signature of two dimensional (2D) diffracting matters. The features at $1.778 \AA^{-1} \leq \mathrm{Q} \leq 1.913 \AA^{-1}$ in the neutron pattern arise from imperfect subtraction of the graphite background. As it can be point out from figure-1, the spectrum measured at the intermediate coverage (1.0 layer) includes peaks observed respectively at 0.7 layer and 1.1 layer. And this will constitute a primary indication that the corresponding phase can be interpreted as a combination of those corresponding to 0.7 and 1.1 layers.

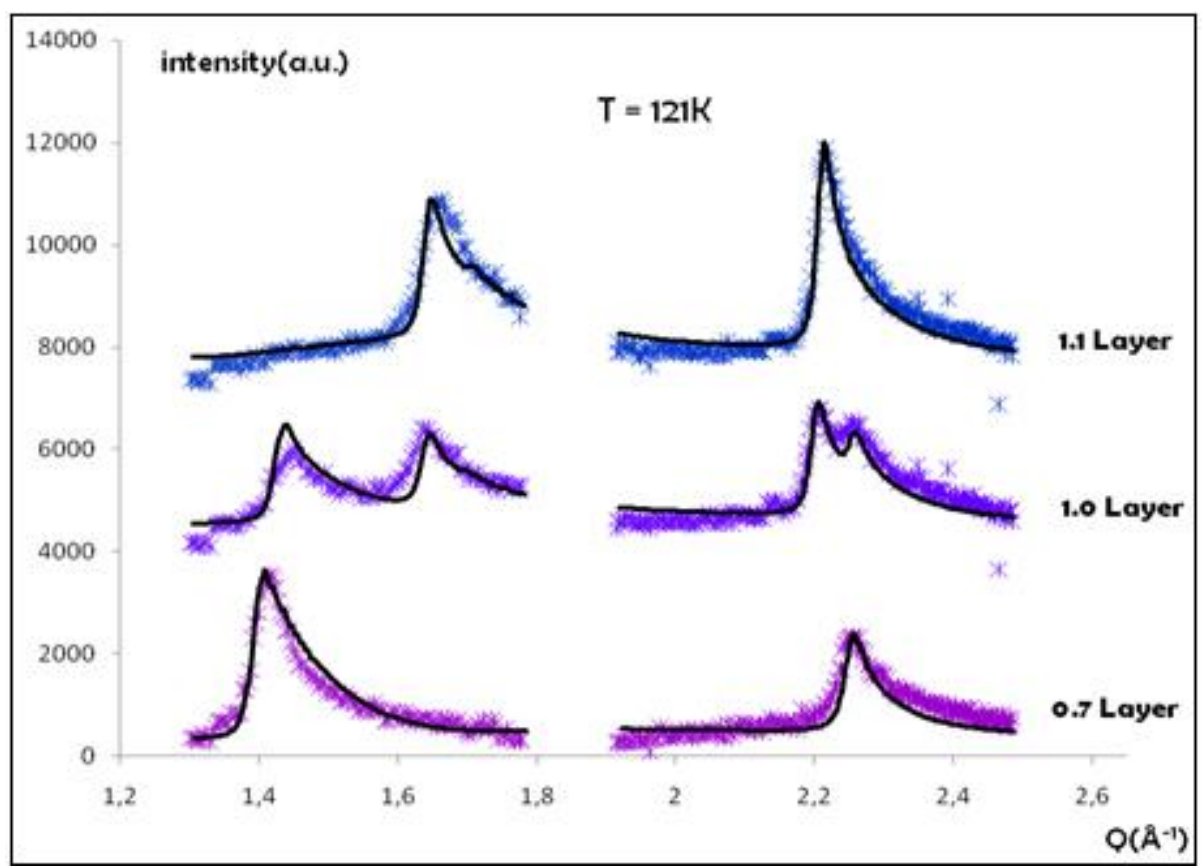

Figure 1: Neutron diffraction spectra by solids $S_{1}$ and $S_{2}$ measured respectively at coverage rates $\theta_{1}=0.7$ layer and $\theta_{2}=1.1$ layer. The intermediate spectrum includes the peaks observed in $S_{1}$ and $S_{2}$. Fits calculated from the parameters given in Table2 are shown in solid lines

Identification of the various (2D) solids

Neutron diffraction spectra by acetylene adsorbed films on graphite were recorded for three different coverages, respectively $\theta_{1}=0.7$ layer, $\theta_{12}=1.0$ layer and $\theta_{2}=1.1$ layers. They are differentiated by the positions of the peaks and their intensities. In Figure-1 we give the three spectra measured at $121 \mathrm{~K}$.

In the following table (Table-1) we give the positions of the peaks deduced from the measured spectra as well as the corresponding inter-reticular distances.
Peak positions given by Thorel et al. are in agreement with those deduced from our spectra. We will see later that the interpretation proposed by Thorel et al. is in agreement with the one we will present although the structures they have proposed seem to us to be incorrect as it will be demonstrated. 


\begin{tabular}{|c|c|c|c|}
\hline$\theta$ (layer) & $\mathrm{Q}_{\left(\AA^{-1}\right)}$ & $\left.\mathrm{Q}_{(} \AA^{-1}\right)[14]$ & $\mathrm{d}(\AA)$ \\
\hline \multirow{2}{*}{0.7} & 1.416 & 1.449 & 4.437 \\
& 2.257 & 2.291 & 2.784 \\
\hline \multirow{3}{*}{1.0} & 1.451 & & 4.330 \\
& 1.636 & & 3.804 \\
& 2.209 & & 2.844 \\
& 2.255 & & 2.786 \\
\hline \multirow{2}{*}{1.1} & 1.662 & 1.648 & 3.780 \\
& 2.217 & 2.244 & 2.834 \\
\hline
\end{tabular}

Table 1: Peaks position and corresponding inter-reticular distances observed in neutron diffraction spectra by twodimensional $\mathrm{C}_{2} \mathrm{D}_{2}$ films adsorbed on graphite at $121 \mathrm{~K}$. The positions of the main peaks observed by Thorel [14] at 57K and for coverage rates close to ours are also given to make comparison easer.

To determine, unambiguously, the parameters $(a, b, \gamma)$ of the unit cell, at least, three peaks are needed. Unfortunately, in the spectra measured at $\theta_{1}$ and $\theta_{2}$ coverages, only two peaks are observed and additional information has to be considered to determine the parameters of the unit cells. The first information comes from the area occupied by a $\mathrm{C}_{2} \mathrm{H}_{2}$ molecule in the $(2 \mathrm{D})$ condensed phase $\left(\boldsymbol{\sigma}_{\mathrm{C} 2 \mathrm{H} 2} \approx 16 \AA^{2}\right)$, determined by comparison of a krypton isotherm to a $\mathrm{C}_{2} \mathrm{H}_{2}$ one, measured separately on the same sample of graphite at approximately equal reduced temperature $T / T_{3 C}$ where $T_{3 C}$ is the bulk critical temperature. The second information considered here is the quadrupolar interaction which induces a $\mathrm{T}$ shaped arrangement of the molecules since the quadrupole moment of $\mathrm{C}_{2} \mathrm{H}_{2}$ is important $\left(\mathrm{Q}=7.2 \mathrm{D} \check{A}^{-1}\right)$. So, only unit meshes containing two molecules and allowing indexation of the observed peaks are retained.

The elementary meshes, whose parameters are given in the table-2, are rectangular centered, they each contain two molecules: one placed at the origin and the other at the center. The positions and orientations of the molecules in the elementary meshs and which made it possible to calculate the best fit of the experimental spectra are summarized in the same table.

For the solid $\mathrm{S}_{1}$, the molecules are slightly straightened and their $\mathrm{C}=\mathrm{C}$ axis are at an angle of $30^{\circ}$ with respect to the surface and are oriented symmetrically with respect to the $\mathrm{x}$ axis.

For the solid $\mathrm{S}_{2}$ the molecules are flat on the surface and make an angle of $90^{\circ}$ between them.

\begin{tabular}{|c|c|c|c|c|c|c|}
\hline $\begin{array}{c}\Theta \\
\text { (layer) }\end{array}$ & $\mathrm{a}(\AA), \mathrm{b}(\AA), \gamma\left({ }^{\circ}\right)$ & $\mathrm{Q}_{\mathrm{hk}}\left(\AA^{-1}\right)$ & $\mathrm{d}_{\mathrm{hk}}(\AA)$ & $\begin{array}{r}\sigma_{\mathrm{C} 2 \mathrm{H} 2} \\
\left(\AA^{2}\right) \\
\end{array}$ & $\mathrm{N} \mathrm{mol} / \mathrm{uc}$ & $\begin{array}{c}\text { Position and orientation } \\
\text { of molecules }(x, y, z, \theta, \varphi)\end{array}$ \\
\hline 0.7 & 9. & $\begin{array}{l}\mathrm{Q}_{20}=1.396 \\
\mathrm{Q}_{21}=2.250\end{array}$ & $\begin{array}{r}\mathrm{d}_{20}=4.50 \\
\mathrm{~d}_{21}=2.792\end{array}$ & 16.02 & 2 & $\begin{array}{c}0 ; 0 ; 4.55 ; 60 ;-70 \\
4.50 ; 1.78 ; 4.55 ; 60 ; 70\end{array}$ \\
\hline 1.0 & \multicolumn{6}{|c|}{$\mathrm{S}_{12}=0.53 \mathrm{~S}_{1}+0.47 \mathrm{~S}_{2}$} \\
\hline 1.1 & $7.36 ; 4.46$ & $\begin{array}{l}\mathrm{Q}_{20}=1.707 \\
\mathrm{Q}_{21}=2.213\end{array}$ & $\begin{array}{l}\mathrm{d}_{20}=3.680 \\
\mathrm{~d}_{21}=2.839\end{array}$ & 16.41 & 2 & $\begin{array}{c}0 ; 0 ; 2.50 ; 90 ; 45 \\
3.68 ; 2.23 ; 3.75 ; 90 ; 135\end{array}$ \\
\hline
\end{tabular}

Table 2: Parameters $(a, b, \gamma)$ of the unit cells proposed for the $\mathrm{C}_{2} \mathrm{D}_{2}(2 \mathrm{D})$ solids formed at $121 \mathrm{~K}$ and for the coverages respectively equal to 0.7 layer and 1.1 layers. These meshes allow indexation of the observed peaks. The positions and orientations of the molecules in the elementary cells are also given: $\theta$ being the angle that the $C=C$ axis of the $C_{2} D_{2}$ molecule form with the vertical to the graphite surface and $\varphi$ the angle between the projection of the $\mathrm{C}=\mathrm{C}$ axis on the plane $(\mathrm{x}, \mathrm{y})$ and the $\mathrm{x}$-axis.

In figure-1, in addition to the recorded spectra (symbols) we give the corresponding fits (continuous line) obtained from the parameters summarized in table-2. For the spectrum measured at a coverage rate equal to 1.0 layer, we noticed that it is formed of the peaks observed at $\theta_{1}=0.7$ layer and those observed at $\theta_{2}=1.1$ layers. This spectrum therefore corresponds to a solid where the two phases $S_{1}$ and $S_{2}$ coexist. We then reproduced this spectrum by combining the spectra corresponding to $S_{1}$ and $S_{2}$. The solid $S_{12}$ is a mixture of $S_{1}$ and $S_{2}\left(S_{12}=0.53 S_{1}+0.47 S_{2}\right)$. It is important, at this level, to note that Thorel et al. have reported that the phase observed for a coverage rate $\theta=0.99$ layer, is the coexistence of the two phases (phase $\alpha$ observed for coverage rates ranging from 0.65 to 0.89 layer and $\beta$ phase for coverage rates ranging from 1.24 to 1.54 layers). Their phase $\alpha$ corresponds to what we denote by $S_{1}$, while their phase $\beta$ corresponds to what we call $S_{2}$. We preferred $S_{1}$ and $S_{2}$ to avoid confusion with the ordered ( $\alpha$ phase) and partially disordered ( $\beta$ phase) phases of bulk acetylene (3D).
In agreement with the study conducted by Thorel et al., the solid $S_{1}$ is stable at least between 0.7 and 0.85 layer which are the two coverage rates at which neutron diffraction spectra were recorded. For the solid $\mathrm{S}_{2}$, the corresponding spectra were recorded for $\theta=1.1$ layers and $\theta=1.23$ layers. What seemed to us inconsistent in the interpretation of Thorel et al. are the structures proposed for solids $S_{1}$ and $S_{2}$ where they have considered a simple single-molecule mesh which means that the quadrupole moments will be aligned, and this seems to be in disagreement with the fact that the $\mathrm{C}_{2} \mathrm{H}_{2}$ molecules are endowed with a strong quadrupole moment $\mathrm{Q}=7.2 \mathrm{D} . \AA^{-1}$. Moreover, by energetic calculations, Anne Terlain [20] has shown that an almost rectangular centered structure is, from energetic point of view, more stable than that proposed by Thorel.

The spectra measured at $\mathrm{T}=133.5 \mathrm{~K}$ and for coverages equal to 0.85 layer and 1.1 layers, corresponding respectively to solids $S_{1}$ and $S_{2}$, have been reproduced by considering the structures mentioned in table- 2 with a slight modification in the parameters (figure-2). And this constitutes additional proof of the validity of the structures proposed for the solids $S_{1}$ and $S_{2}$ on the one hand and of the invariance of the structures when the temperature increases on the other hand, ie a strong index of the commensurability of each of the structures (2D) with the surface (0001) of graphite. 


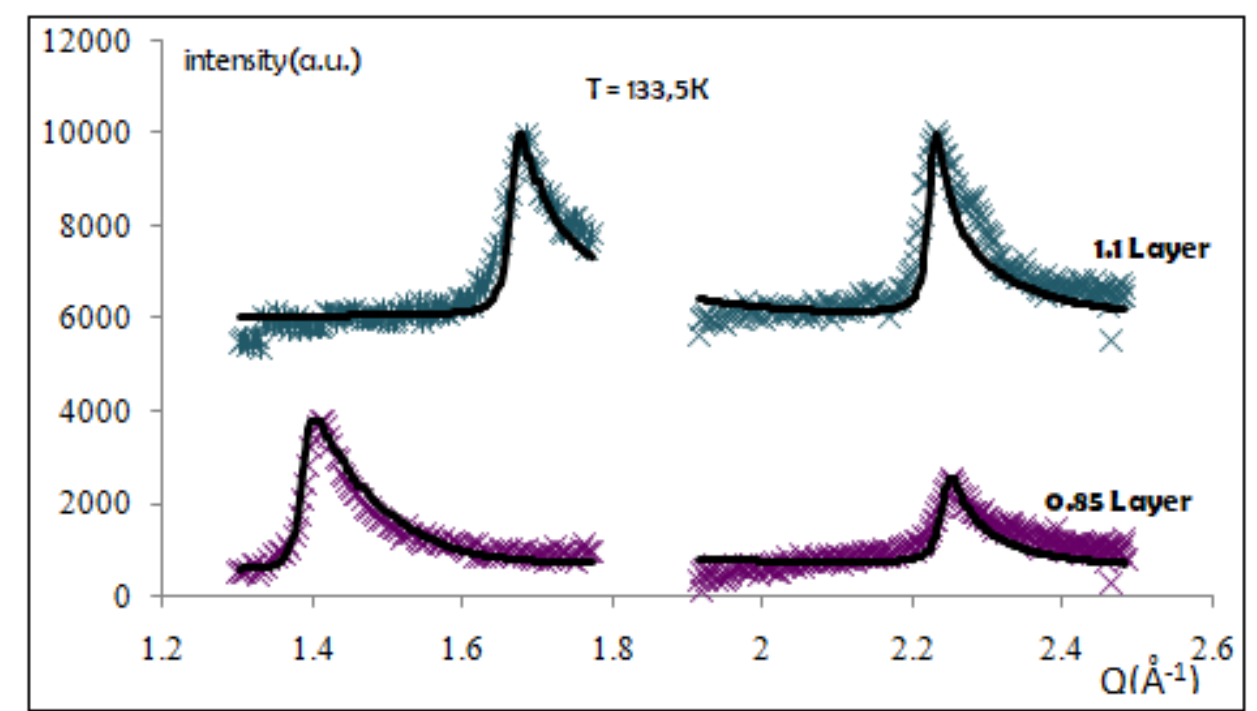

Figure 2: Neutron diffraction spectra by two different coverages and corresponding to $S_{1}$ and $S_{2}$ phases, measured at $T=$ $133.5 \mathrm{~K}$

A schematic representation of the elementary cells is given in Figure-3, below.

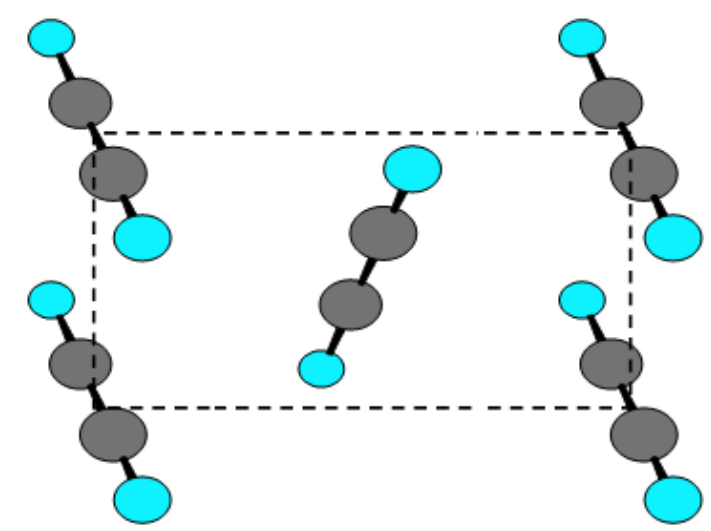

Figure 3 (a)
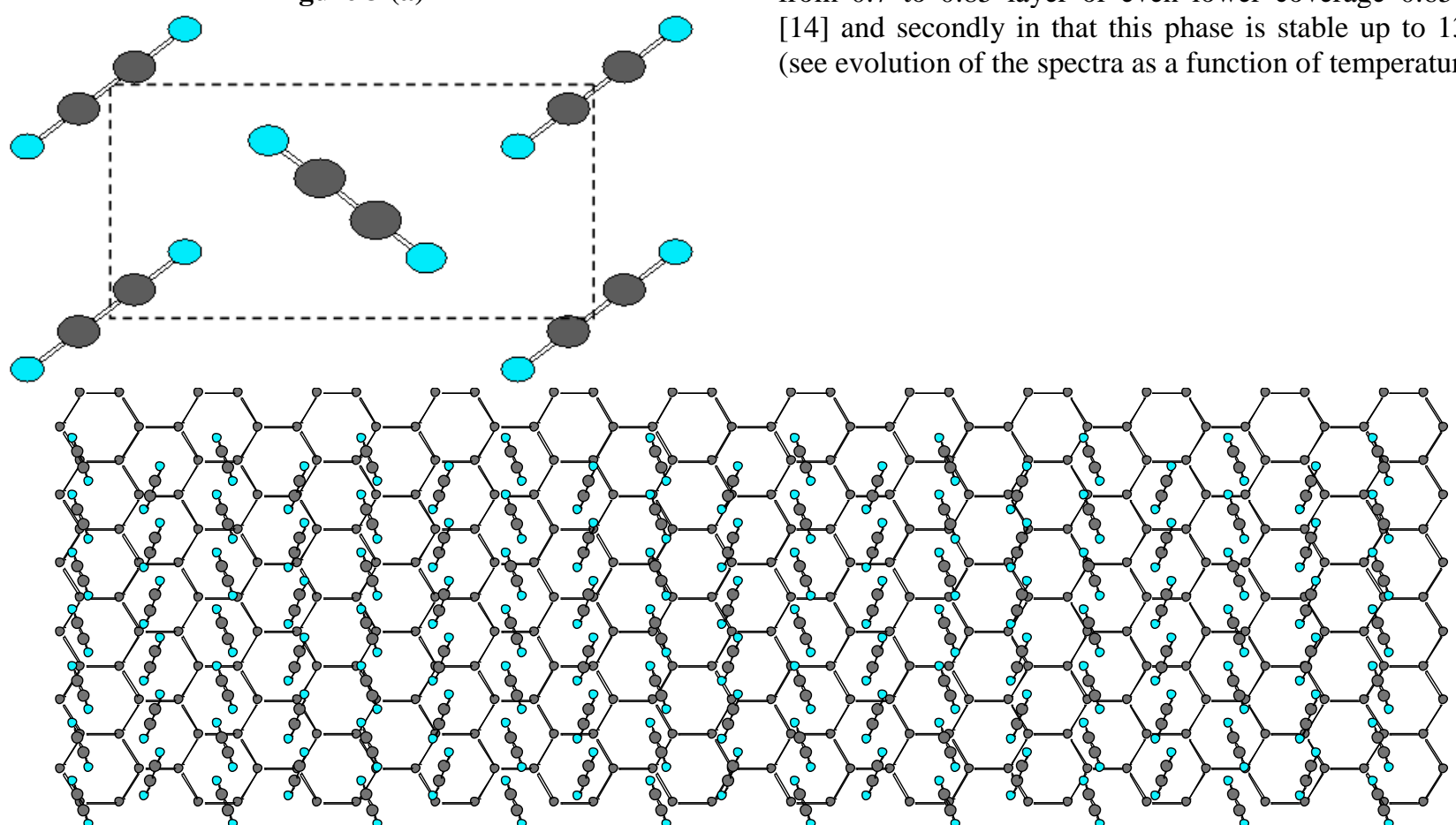

Figure 4: Solid $\mathrm{S}_{1}$ : commensurable mesh containing 108 molecules of $\mathrm{C}_{2} \mathrm{D}_{2}$
Figure 3 (b)

Figure 3: Schematic representation of the two elementary cells at $T=121 \mathrm{~K}(\mathrm{a})$ : solid $\mathrm{S}_{1}$, (b) solid $\mathrm{S}_{2}$.

The reproduction of the elementary cell of the solid $S_{1}$ (figure-4) shows that this solid phase has commensurability at a great distance with the (0001) surface of the graphite. In matrix notation, this commensurability is given by

$$
\left(\begin{array}{l}
a_{a d s} \\
b_{a d s}
\end{array}\right)=\left(\begin{array}{cc}
33 & 0 \\
5 & 10
\end{array}\right)\left(\begin{array}{l}
a_{s u b} \\
b_{s u b}
\end{array}\right) \text { where } a_{s u b}=b_{s u b}=2.46 \AA \text {. }
$$

The commensurable cell contains 108 molecules of $\mathrm{C}_{2} \mathrm{D}_{2}$. This commensurability is supported on the one hand by the fact that the solid $S_{1}$ is stable for a coverage rate ranging from 0.7 to 0.85 layer or even lower coverage 0.65 layer [14] and secondly in that this phase is stable up to $133.5 \mathrm{~K}$ (see evolution of the spectra as a function of temperature). 
In matrix notation, it presents the commensurability

$$
\left(\begin{array}{l}
a_{a d s} \\
b_{a d s}
\end{array}\right)=\left(\begin{array}{cc}
33 & 0 \\
5 & 10
\end{array}\right)\left(\begin{array}{l}
a_{s u b} \\
b_{s u b}
\end{array}\right) \text {. }
$$

The location of the $\mathrm{C}_{2} \mathrm{D}_{2}$ molecules centers on the (0001) graphite surface, as in the following figure, is arbitrary. Low energy electron diffraction manipulations as well as energy minimization calculations will give the epitaxy of the films with the graphite surface.

Similarly, the reproduction of the elementary cell of the solid $\mathrm{S}_{2}$ (figure-5) shows that this solid phase present an original commensurability with the (0001) face of the graphite. In matrix notation, this commensurability is given as $\left(\begin{array}{l}a_{a d s} \\ b_{a d s}\end{array}\right)=\left(\begin{array}{ll}7 & 2 \\ 4 & 11\end{array}\right)\left(\begin{array}{l}a_{s u b} \\ b_{s u b}\end{array}\right)$, the commensurable cell contains 22 molecules of $\mathrm{C}_{2} \mathrm{D}_{2}$. This commensurability is consolidated on the one hand by the fact that the solid $\mathrm{S}_{2}$ is stable for a coverage rate ranging from 1.1 to 1.23 layers or even 1.54 layers [14] and secondly by the fact that this phase is stable up to $141 \mathrm{~K}$, the highest temperature at which we recorded a diffraction spectrum for this coverage rate (see evolution of the spectra as a function of temperature).

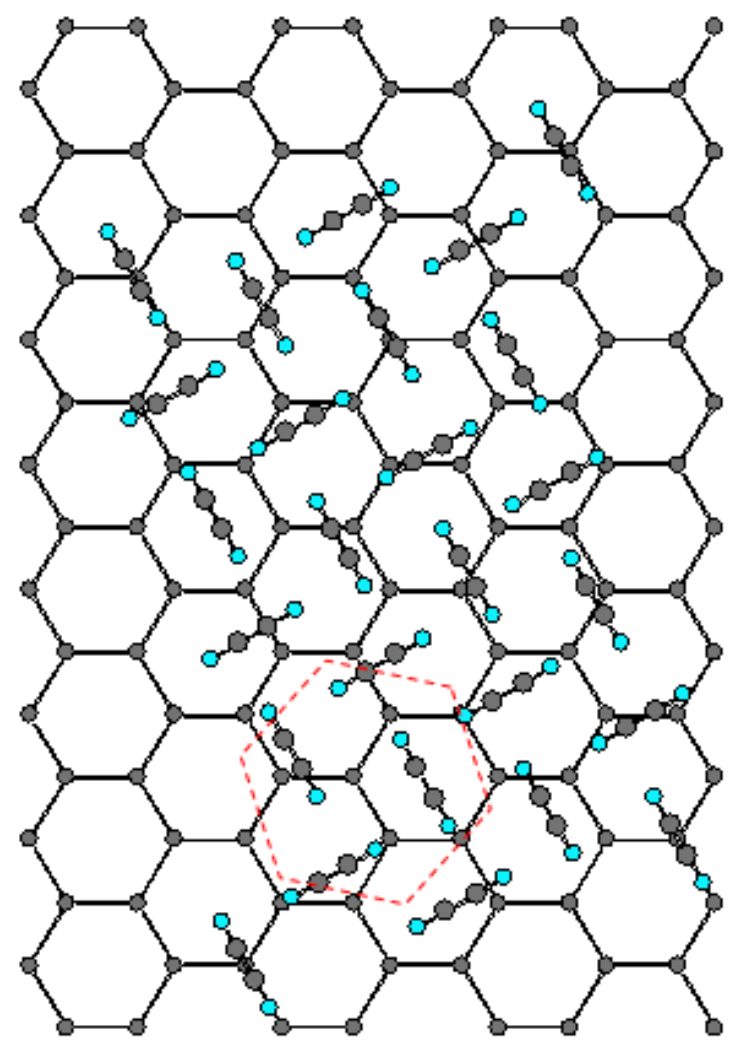

Figure 5: Solid $\mathrm{S}_{2}$ : commensurable cell containing 22 molecules of $\mathrm{C}_{2} \mathrm{D}_{2}$. In matrix notation, it presents the

$$
\text { commensurability: }\left(\begin{array}{l}
a_{a d s} \\
b_{a d s}
\end{array}\right)=\left(\begin{array}{ll}
7 & 2 \\
4 & 11
\end{array}\right)\left(\begin{array}{l}
a_{s u b} \\
b_{s u b}
\end{array}\right)
$$

In this phase, $S_{2}$, and as it can be shown in figure-5, the centers of mass of the admolecules are organized in an

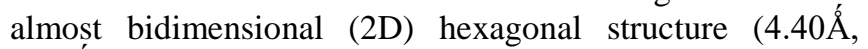
$4.40 \AA \hat{A}, 117.57^{\circ}$ ). A slight increase in the coverage (from 1.1 to 1.23 layers) does not change the structure of the solid but the parameters of the mesh are slightly modified, leading the centers of mass of the admolecules to organize themselves in

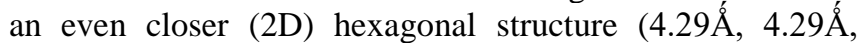
$\left.118.62^{\circ}\right)$. This comportment is illustrated in table-3 where the evolution of the parameters of the rectangular centered cells according to the coverage rate is given. It seems that this evolution will continuous until the structure of the $S_{2}$ solid reaches a strong commensurability $\sqrt{3} x \sqrt{3}$ with the (0001) surface of graphite. But, an increase in the coverage must be accompanied by at least partial overlap of the molecules so that they keep the same structure. The calculated spectra of the $S_{2}$ solid show that the adsorbed molecules remain parallel to the surface independently of the coverage and the compression of the film is manifested at the level of the admolecules height by a slight variation of their position along the $\mathrm{z}$-axis.

Table 3: Evolution of the parameters of the elementary meshes: for $\mathrm{T}=121 \mathrm{~K}$ and for $\mathrm{T}=133.5 \mathrm{~K}$. Solids $\mathrm{S}_{1}, \mathrm{~S}_{2}$ are

\begin{tabular}{|c|c|c|c|c|c|c|}
\hline \multirow{2}{*}{$\begin{array}{c}\text { Temperature } \\
(\mathrm{K})\end{array}$} & \multirow[t]{2}{*}{ solid } & \multirow[t]{2}{*}{$\begin{array}{c}\text { Coverage } \\
\text { (layer) }\end{array}$} & \multicolumn{3}{|c|}{$\begin{array}{l}\text { Parameters of } \\
\text { the unit mesh }\end{array}$} & \multirow{2}{*}{$\begin{array}{l}\text { average area occupied } \\
\text { by a molecule } \sigma\left(\AA^{2}\right)\end{array}$} \\
\hline & & & $\mathrm{a}(\AA)$ & $\mathrm{b}(\AA)$ & $\gamma\left({ }^{\circ}\right)$ & \\
\hline \multirow[b]{2}{*}{133.5} & \multirow{2}{*}{$\mathrm{S}_{1}$} & 0.7 & 9.04 & 3.56 & 90 & 16.09 \\
\hline & & 0.85 & 9.02 & 3.56 & 90 & 16.05 \\
\hline \multirow[b]{2}{*}{121} & \multirow[b]{2}{*}{$\mathrm{S}_{2}$} & 1.1 & 7.36 & 4.46 & 90 & 16.41 \\
\hline & & 1.23 & 7.38 & 4.38 & 90 & 16.16 \\
\hline
\end{tabular}
stable in a broad overlap rates.

Evolution of neutron diffraction spectra as a function of temperature:

a) Solid $\mathrm{S}_{1}(0.7 \leq \theta \leq 0.85$ layer $)$

The evolution of the diffractograms recorded at $\theta=0.7$ layer and $\theta=0.85$ layer as a function of the temperature are represented in Figs. 6 and 7 respectively. These spectra are attributed to the solid $S_{1}$, they have two main peaks which undergo a slight displacement when the temperature increases. This displacement reflects a small expansion of the mesh when the temperature increases. They disappear beyond $133,50 \mathrm{~K}$, leaving their place to two rather large bumps, which are the signature of a liquid phase. It follows that the solid $S_{1}$ has a melting temperature $T_{m}\left(S_{1}\right)$ of the order of $135 \pm 1 \mathrm{~K}$. 


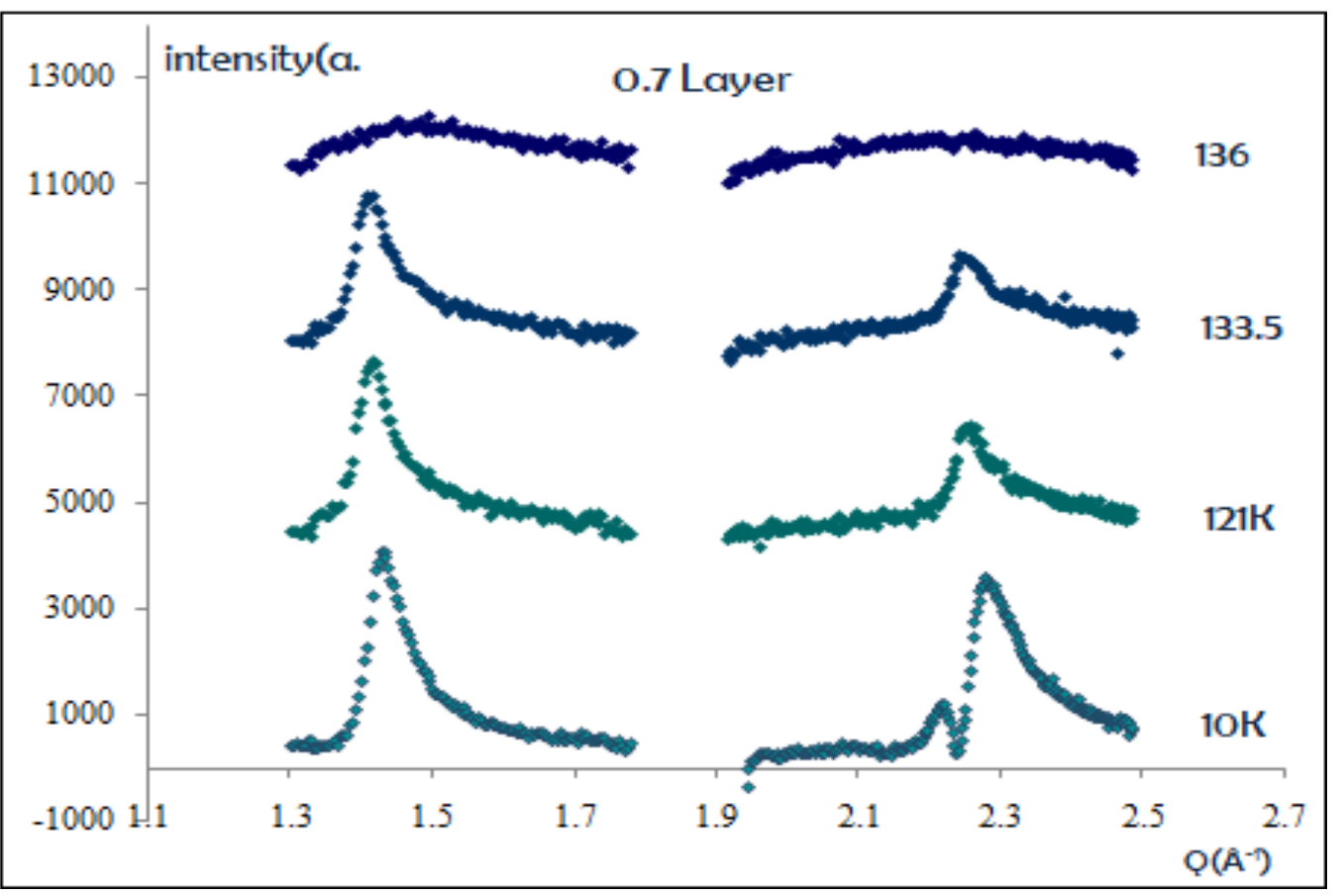

Figure 6: Evolution of the neutron diffraction spectra by 0.7 layer of $\mathrm{C}_{2} \mathrm{D}_{2}$ adsorbed on graphite as a function of temperature. The fusion temperature of solid phase $S_{1}$ was estimated to be about $135 \pm 1 \mathrm{~K}$.

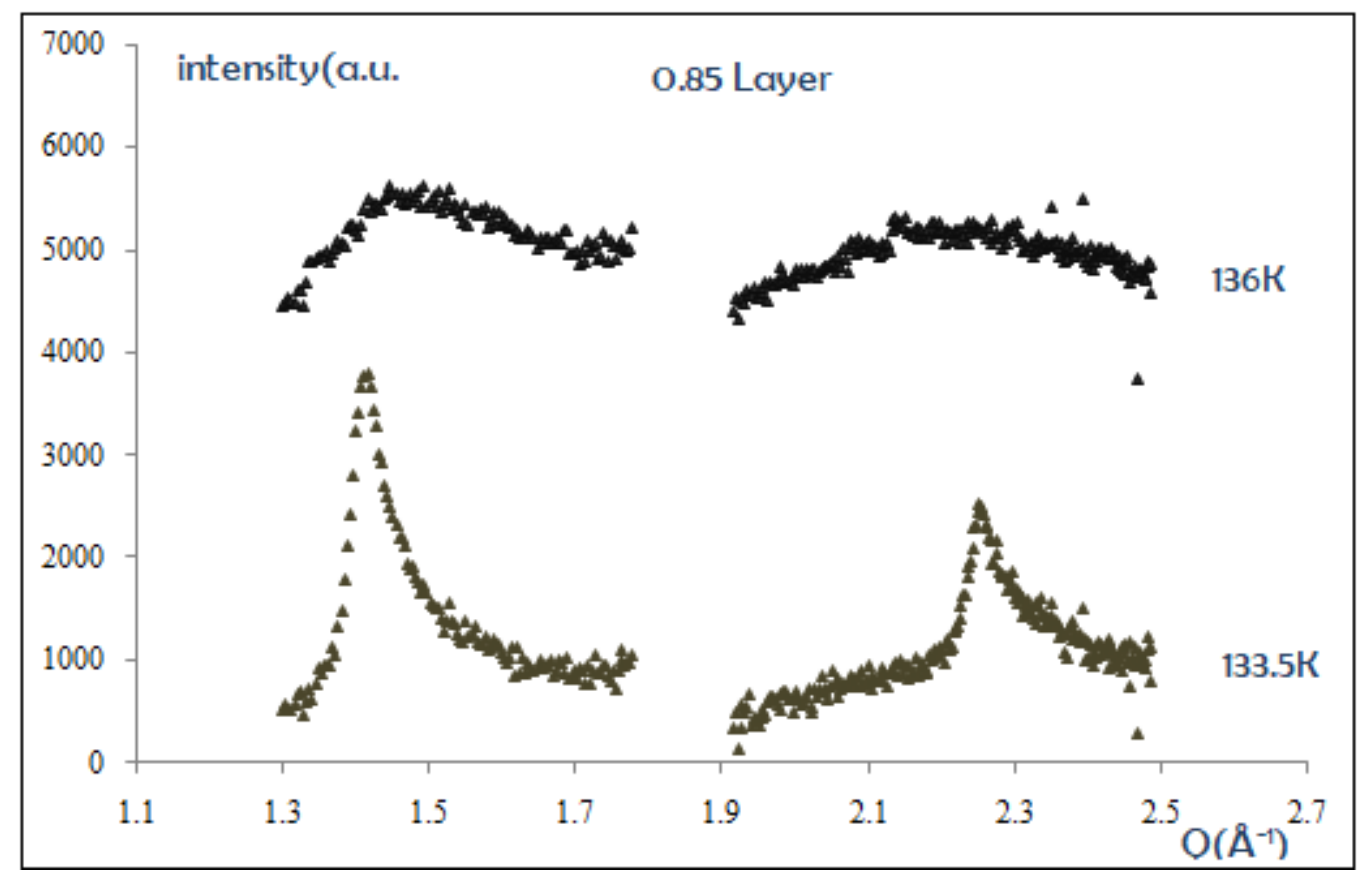

Figure 7: Evolution of the neutron diffraction spectra by 0.85 layer of $\mathrm{C}_{2} \mathrm{D}_{2}$ adsorbed on graphite as a function of temperature

b) Solid $S_{2}(1.1 \leq \theta \leq 1.23$ layers $)$

The evolution of the neutron diffraction spectra as a function of temperature by the coverage rates of 1,1 layers and 1,23 layers is shown in Figs. 8 and 9 respectively. The peaks keep their dissymmetric forms even at $141 \mathrm{~K}(\theta=1.23$ layers $)$, thus indicating that this solid $\mathrm{S}_{2}$ has a melting point $\mathrm{T}_{\mathrm{m}}\left(\mathrm{S}_{2}\right)$ greater than $141 \mathrm{~K}$. It is found that $\mathrm{T}_{\mathrm{m}}\left(\mathrm{S}_{2}\right)>\mathrm{T}_{\mathrm{m}}\left(\mathrm{S}_{1}\right)$ and this is in agreement with the fact that $S_{2}$ present a stronger commensurability with the surface $(0001)$ of the graphite compared to that of the solid $\mathrm{S}_{1}$. 


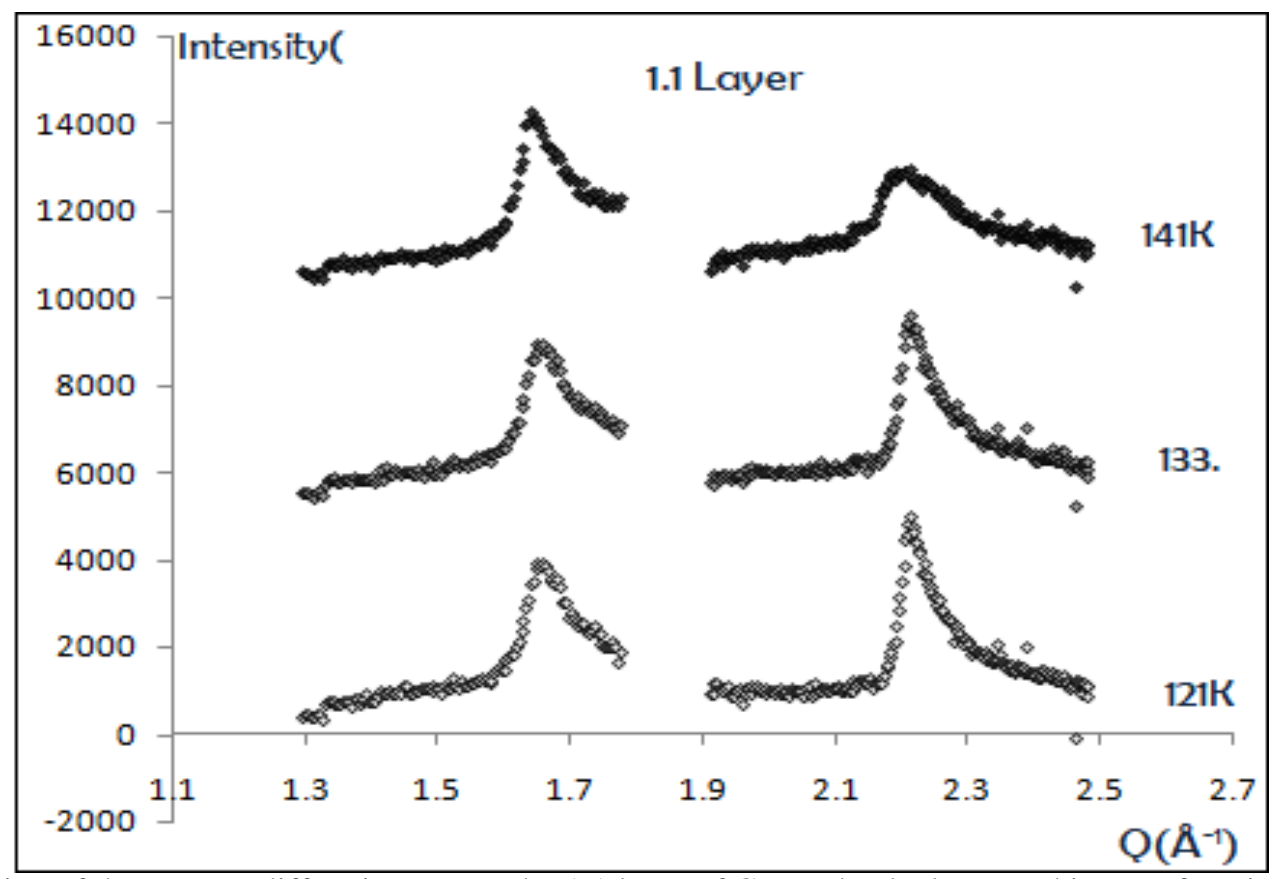

Figure 8: Evolution of the neutron diffraction spectra by 1.1 layer of $\mathrm{C}_{2} \mathrm{D}_{2}$ adsorbed on graphite as a function of temperature. Even at $141 \mathrm{~K}$, the peaks are well defined suggesting the condensed phase to be solid rather than liquid. The solid $\mathrm{S}_{2}$ melts at $\mathrm{T}>141 \mathrm{~K}$.

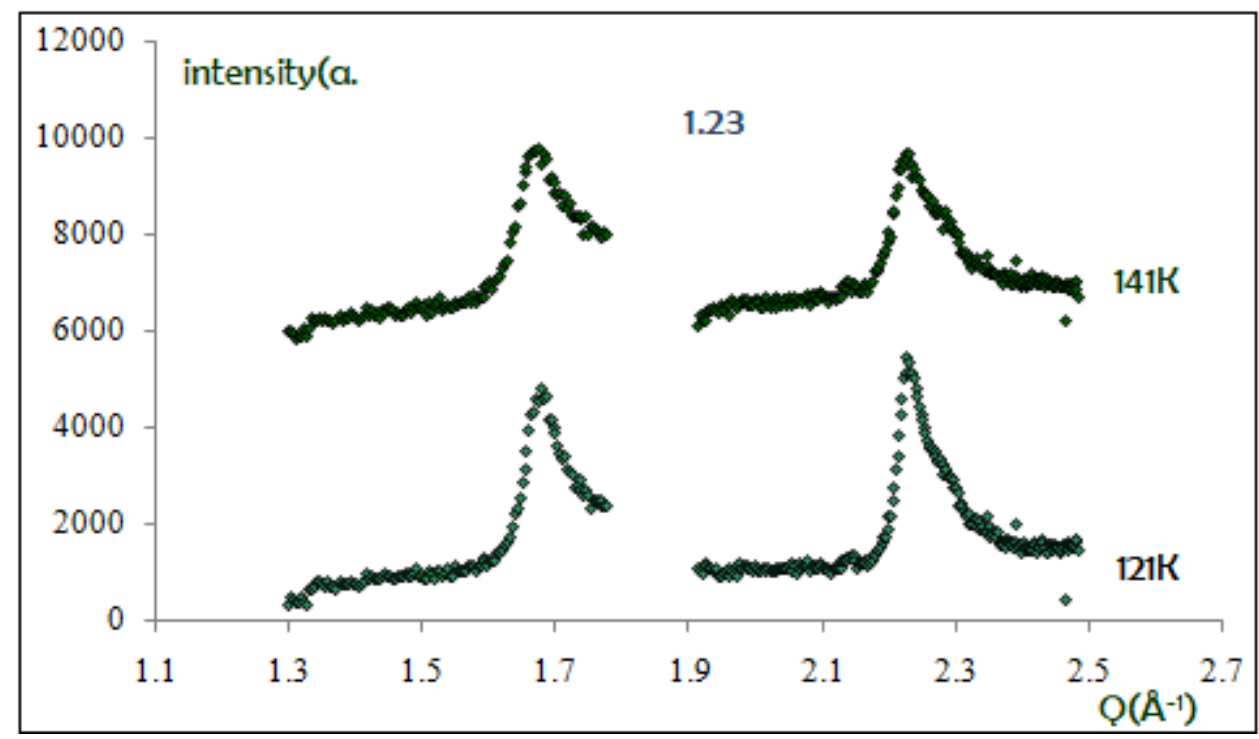

Figure 9: Evolution of the neutron diffraction spectra by 1.23 layer of $\mathrm{C}_{2} \mathrm{D}_{2}$ adsorbed on graphite as a fonction of temperature. The solid character of the adsorbed phase is evidenced even at $141 \mathrm{~K}$.

\section{c) Solid $S_{12}(\theta=1.0$ layer $)$}

For this coverage rate, the evolution of the spectra as a function of temperature is given in Figure-10. The spectra measured at $121 \mathrm{~K}$ and $133.50 \mathrm{~K}$ show four peaks where each pair characterizes one of the two solids $S_{1}$ and $S_{2}$. Beyond $133.5 \mathrm{~K}$, only the characteristic peaks of $S_{2}$ persist. Indeed, the peaks relative to the solid $S_{1}$ disappear beyond the melting temperature $T_{m}\left(S_{1}\right)$. The fact that in the spectra measured at $136 \mathrm{~K}$ and $141 \mathrm{~K}$, the peaks are less intense and poorly defined does not involve the melting of the $\mathrm{S}_{2}$ solid at a temperature below $141 \mathrm{~K}$. In fact, once the domains corresponding to the solid $S_{1}$ are melted, the molecules that constituted them will find a mobility leading them to partially cover the domains corresponding to the solid $S_{2}$ thus blurring their signal. 


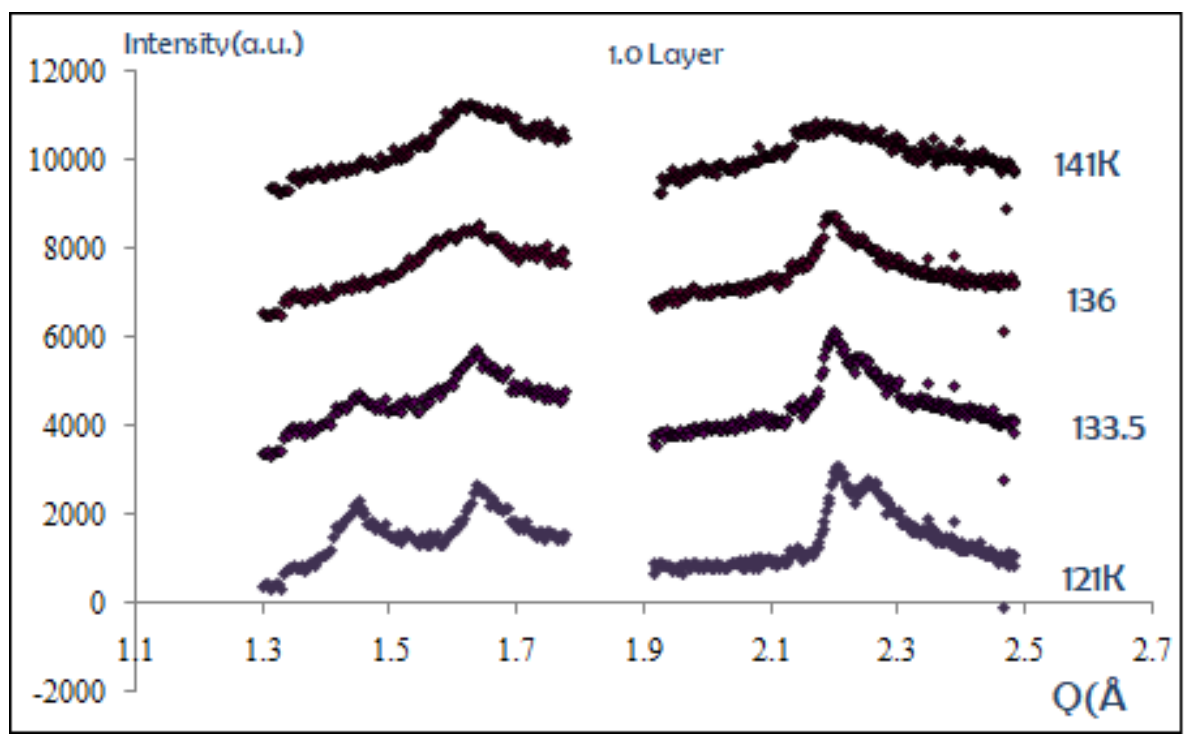

Figure 10: Evolution of the neutron diffraction spectra by 1.0 layer of $\mathrm{C}_{2} \mathrm{D}_{2}$ adsorbed on graphite as a function of temperature. The peaks relatif to the $\mathrm{S}_{1}$ solid phase disappear between $133.5 \mathrm{~K}$ and $136 \mathrm{~K}$.

\section{Conclusion}

Neutron diffraction technique has been used to determine structures of the (2D) deuterated acetylene adsorbed phases on graphite. Spectra have been measured at coverages ranging from 0.7 to 1.23 layers. Two bidimensional (2D) solid phases have been identified. The structure of the solid $\mathrm{S}_{1}$ which is stable at a wide range of coverage ( 0.65 to 0.85layer) present long range commensurability with the (0001) face of graphite. This commensurability is also supported by the fact that the corresponding structure was stable from $10 \mathrm{~K}$ to $133.5 \mathrm{~K}$. The fusion of $\mathrm{S}_{1}$ occurs at $\mathrm{T}_{\mathrm{m}}$ $\left(\mathrm{S}_{1}\right)=135 \pm 1 \mathrm{~K}$. The structure of the solid $\mathrm{S}_{2}$ corresponding to a coverage $\geq 1.1$ layer present an original commensurability with the (0001) surface of graphite. This commensurability was supported on one hand by the fact that it is stable from 1.1 layer to 1.54 layer and on the other hand by the fact that is stable at temperature $>141 \mathrm{~K}$, the upper temperature at which a spectrum has been measured. So $\mathrm{T}_{\mathrm{m}}\left(\mathrm{S}_{2}\right)>141 \mathrm{~K}$. An other originality, characterizing the $\mathrm{C}_{2} \mathrm{D}_{2}$ /graphite system comes from the (2D) solid phase corresponding to a coverage equal to 1.0 layer and which was identified as a mixture of $S_{1}$ and $S_{2}$ solids. A similar situation has been encountered when studying the adsorption of $\mathrm{C}_{2} \mathrm{D}_{6}$ on $\mathrm{MgO}$ (100) [21]. Finally we can conclude that this structural characterization, coupled to a previous thermodynamic one constitute an important data to elaborate phase diagram of the two dimensional matter of $\mathrm{C}_{2} \mathrm{D}_{2}$ adsorbed on graphite.

\section{References}

[1] Thomas Arnold, Richard E. Cook, Sami Chanaa, Stuart M. Clarke, Michael Farinelli, Peter Yaron, J.Z. Larese. Physica B 385-386 (2006)205-205.

[2] T. Arnold, A. Barbour, S. Chanaa, R.E. Cook, D. Fernandez-Canato, P. Laudry, T. Seydel, P. Yaron and J.Z. Larese. The Europran Physical Journal Special Topics, 167, 143-150 (2009).

[3] M. Bienfait, P. Zeppenfield, N. Dupont-Pavlowsky, M. Muris, M.R. Johnson, T. Wilson, M. DePies and O.E. Vilches. Physical Review B 70, 035410 (2004).
[4] V. Goudon, J.C. Lasjaunias Adsorption (2008) 14, 1-9.

[5] L.W. Bruch, J.Z. Larese Physical Review B 85, 035401 (2012).

[6] Zsuzsanna E. Mészar, György Hantal, Sylvain Picaud and Pal Jedlovszky J. Phys. Chem. C 2013, 117, 6719-6729.

[7] D. Fernandez-Cacoto and J.Z. Larese. J. Phys. Chem. C $\mathrm{xxxx}, \mathrm{xxx}, \mathrm{xxx}-\mathrm{xxx}$.

[8] M. Ben Yahya, F. Aouaini, M.A. Hachicha, M. Khalfaoui, A. Ben Lamine. Physica B 419 (2013) 100-104.

[9] Thomas Arnold, Stuart M. Clarke. Current Opinion in Colloid \& Interface Science 17 (2012) 23-32.

[10] Shuhui Cai, Konstantin M. Neyman, Helmut Knözinger, Notker Rösch. Surface Science 479 (2001) 169-182.

[11] D. Ferry, J. Suzanne Surface Science 345 (1996) L19-L22.

[12] D. Ferry, J. Suzanne, P.N.M. Hoang, C. Girardet. Surface Science 375 (1997) 315-330.

[13] J. Menaucourt, A. Thomy and X. Duval. Journal de chimie physique, $1980,77, \mathrm{n}^{\circ} 10$

[14] P. Thorel, C. Marti, G. Bomchil and J.M. Alloneau. Fourth International Conference on Solid Surface and the Third European Conference on Surface Science, Cannes 1980, Eds D.A. Degras et M. Costa, Société Française du vide, Paris, Vol. 1 p119.

[15] C. Peters, J.A. Morrison and M.L. Klein. Surface Science 165 (1986) 355-374.

[16] M. Trabelsi and Y. Larher. Surface Science Letters 275 (1992) L631-L635.

[17] S. Keith Dunn and G.E. Ewing. J. Vac. Sci. Technol. A 11 (4) 1993.

[18] F. Khémiri, M. Trabelsi, S. Saidi, J.P. Coulomb and Y. Larher. Indian Journal of Pure \& Applied Physics. Vol.53, September 2015, pp. 612-616.

[19] Yosra Ben Torkia, Wouroud Sghair, Mohamed Bouzid, Mohsen Trabelsi, Abdelmottaleb Ben Lamine International Journal of Polymer Analysis and Characterization (in press)

[20] A.Terlain, thèse

[21] Structural study of $\mathrm{C}_{2} \mathrm{D}_{6}$ thin films adsorbed on $\mathrm{MgO}$ (100). Indian Journal of Pure \& Applied Physics, Vol. 53, November 2015, pp. 748-752. S. Saidi , M. Trabelsi, C. Chefi \& J.P. Coulomb 